\title{
L'EDUCATION
}

\section{DU CITOYEN}

L'éducation du citoyen n'a pas à former le militant politique. Elle ne doit pas non plus se contenter d'informer l'usager des services publics ou le client des administrations. Dans les limites d'une pratique réaliste, elle se conçoit comme une éducation du jugement fournissant aux élèves les critères formels du droit. Elle s'appuie sur les droits de l'homme en évitant de les transformer en un nouveau catéchisme. Elle noue la réflexion à l'action en visant à faire de l'idée de droit une véritable force politique.

Il me semble intéressant de poser la question de l'éducation du citoyen du point de vue de celui qui est en charge de cette éducation, de celui qui a pour tâche de la mener dans le cadre des instituions scolaires. Il peut en effet se demander quel sens peut avoir cette obligation qui lui est faite.

L'obligation d'éduquer le citoyen, en effet, est quelque peu embarrassante. Notre embarras est symptomatique de la situation qui est la nôtre. Car, d'une part, nous sommes imprégnés d'une sorte de méfiance à l'égard des pouvoirs, de réticence à l'égard de l'Etat, et nos habitudes nous amenent plutôt à cultiver une sorte de distance, de recul ironique à l'égard des institutions. Nous ne croyons pas au caractère sacrée des lois parce que nous savons que les lois servent des intérêts qui ne sont pas forcément les nôtres, et nous sommes soucieux de ne pas montrer une complaisance coupable à l'égard des pouvoirs, de ne pas faire preuve d'une croyance inconsidérée aux vertus mythiques de l'Etat. Il nous semble que la liberté individuelle, si le mot doit avoir un sens, se joue aux marges du système, dans ses interstices ou ses failles, mais certainement pas en accordant un sens positif à la vie dans l'Etat et une signification à la notion d'autorité politique. Mais, d'un autre côté, nous voyons bien que nous sommes confrontés à des problèmes qui ne se résoudront pas tout seuls ou par l'intervention d'un homme providentiel, La montée des racismes, l'exacerbation des intégrismes, le regain de la violence politique, l'abandon du respect des personnes, nous font bien sentir qu'il y a des problèmes à l'égard desquels cette distance ironique 
à l'égard des pouvoirs esr une réponse insuffisante. Il faut tenter d'imaginer autre chose, non en renonçant à l'ironie et à la critique, mais en imaginant quelque chose de plus. C'est la raison pour laquelle nous sommes obligés de prendre au sérieux la question de l'éducation des citoyens : nous sentons bien que nous ne pouvons nous dispenser d'éduquer ceux qui, par leur vote, peuvent engager la communauté sur des voies détestables.

Peut-être une bonne manière de formuler cette question est-elle d'être attentif à l'ambiguité du terme d'éducation en général, ou plus précisément à la double définition qu'on peut en donner. D'une part, en effet, l'éducation peut être conçue du point de vue de la communauté, comme un processus de reproduction de la société qui, pour parler comme Durkheim, n'a pas pour objet essentiel l'individu mais d'inculper aux jeunes générations les valeurs qui font l'unité de la société et lui permettent ainsi de se perpétuer elle-même. Sur le plan proprement politique, l'éducation est ce processus par lequel l'Etat se donne les moyens de sa propre conservation en inculquant aux jeunes générations les vảleurs sur lesquelles il est fondé. Mais, de surcroît, l'Etat est obligé d'exiger des citoyens un certain nombre de valeurs fondamentales, qui tiennent à cette conservation mais aussi au fait que cet Etat n'est pas tout seul, puisqu'il est confronté à un certain nombre de menaces ou de rivalités extérieures et qu'il doit se soucier de sa propre puissance. Il exige ainsi des citoyens des qualités telles que l'obéissance aux lois, le respect des institutions, la loyauté à l'égard de la communauté, et peut-être quelque chose comme un souci de l'intérêt général. On voit bien en quoi cette perspective éducative fait problème à un autre type de point de vue: un point de vue plus conforme à la tradition moraliste et plus centré sur l'individu. L'éducation, dans cette autre perspective, consiste en un processus d'humanisation. Elle consiste à donner à l'individu les moyens de la liberté en le conduisant à l'autonomie. Les deux perspectives, au moins sous un aspect superficiel, sont donc opposées. Dans la pratique, tout au moins, elles ne se concilieront pas sans difficulté.

Il est clair que cette conciliation n'est possible qu'en concevant l'Etat comme l'organisation institutionnelle des libertés. Dans un tel Etat, l'éducation du citoyen, si elle le mène à la responsabilité, à l'exercice de l'autonomie dans la participation à la vie de la communauté, est par làmême une éducation morale.

Tout le probleme est d'essayer de concevoir ce que peut bien être l'organisation institutionnelle des libertés. On peut bien entendu refuser la question et postuler qu'il n'y a pas de liberté. Mais si, par hypothèse, l'expression a un sens, on peut essayer de voir à quelles 
conditions elle a un sens. Si l'on se tourne du côté de la pensée du XVIIIe siècle, et particulièrement du côté de Rousseau, on peut imaginer deux conditions auxquelles le citoyen jouit d'une sorte de liberté politique. Première condition: il est soumis à des lois, c'est-à-dire à l'autorité de la loi, et non au pouvoir personnel d'un individu ou d'un groupe d'individus. Il est libre dans la mesure où il n'est soumis qu'à l'aurorité impersonnelle de la loi, et soustrait au pouvoir d'un ou de plusieurs maîtres. Je ne suis libre, en d'autres termes, que si le lieu du pouvoir est vide, occupé uniquement par un principe normatif qui n'appartient à personne. Et d'autre part, je suis libre - c'est la seconde condition -, si la loi est l'expression de la volonté générale. Celle-ci étant par définition la mienne, en obéissant à la volonté générale je n'obéis qu'à moi-même - ce qui est la définition de la liberté.

Cette théorie est extrêmement séduisante, mais on voit bien où est la difficulté. Elle tient au fait que la loi n'est pas impersonnelle, d'une part, et d'autre part qu'elle n'est pas l'expression de la volonté générale. Elle n'est pas impersonnelle parce qu'elle est le résultat d'un certain nombre de compromis où s'expriment les rapports de force entre les différentes constituantes de la société. Elle n'est pas générale, parce qu'elle n'émane pas d'une multiplicité d'avis purement individuels. En effet, seule une volonté se dégageant d'une telle multiplicité serait véritablement générale. Dès qu'il existe des partis, la loi n'est plus l'expression de la volonté générale au sens propre du terme, elle est l'expression de la volonté des partis ou du consensus qu'ils sont parvenus à établir entre eux. La théorie doit donc subir de sérieuse entorses pour s'adapter à la réalité. D'autant que le modèle rousseauiste veut que le pouvoir exécutif soit l'exécutant des lois, c'est-à-dire que le lieu de la souveraineté soit précisément le législatif, la volonté générale à proprement parler. Or, nous savons très bien que, dans le fonctionnement rél des Etats modernes, les choses ne se passent pas de cette manière. C'est l'exécutif qui est le pouvoir prépondérant. Et, loin qu'il se borne à exécuter les lois, c'est lui qui a l'initiative de la majorité d'entre elles. Il ne se contente pas d'exécuter les lois, celles-ci sont l'expression juridique de ses initiatives.

Par conséquent, la liberté politique conçue dans cette perspective est difficile à maintenir dans toute son intégrité, puisqu'elle suppose une participation aussi directe et constante que possible à l'activité législative. On peut donc être tenté de se replier sur une position plus modeste et plus réaliste, selon laquelle la liberté politique consiste à jouir de la protection des lois, à travailler paisiblement en veillant à son propre bienêtre, à mener une existence privée à l'abri d'un système d'institutions, 
tandis que, pour le reste, la gestion des affaires est confiée à une minorité de professionnels de la politique ou au souci des hommes compétents. Nous sommes ainsi devant une sorte d'alternative, entre le rêve ou l'utopie d'une liberté proprement individuelle, de la liberté de celui qui trouve sa place dans un système qui, par hypothèse, lui garantit une certaine sécurité. Dans cette dernière optique, nous avons affaire à une liberté nécessairement passive sur le plan politique. Sans compter qu'elle est pour beaucoup une liberté formelle. Qu'est-ce que la liberté de travailler en paix ou de jouir de l'existence dans un cadre privé? Est-ce que tout le monde en jouit de la même manière? Cela n'est pas évident. Aussi ce deuxième type de liberté politique cache-t-il bien des inégalités qui ne s'avouent pas.

Sur le plan pédagogique, et pour revenir à l'éducation du citoyen proprement dite, cette alternative peut être traduite de la manière suivante. Ou bien nous envisageons la liberté politique comme participation active de tous, et dans ce cas il faut donner une information très complète aux enfants, de même que la possiiblité, les aptitudes, la clairvoyance et les habitudes qui sont nécessaires à l'activité politique. Dans cette ligne, on rejoindrait certaines utopies révolutionnaires - je pense par exemple au rapport Bouqier (24 Germinal AN II), qui voulait que les sociétés populaires devinssent de véritables lycées républicains, dans l'idée que l'éducation du citoyen devait se faire en amenant les enfants à assister à des assemblées populaires, à des débats, à observer comment les décisions se prennent. L'autre branche de l'alternative consiste à dire qu'après tout il s'agit de former des travailleurs honnêtes, des individus cherchant pacifiquement leur propre bonheur. On peut, des lors, se contenter d'un enseignement minimal: instruire chacun de ses droits et de ses devoirs, et pour le reste apprendre aux enfants les gestes élémentaires de la vie sociale: gérer un budget, signer un chèque, etc.

Pour essayer de donner un sens à cette notion d'éducation du citoyen, si l'on estime que cette alternative n'est pas satisfaisante, il faut tenter de trouver une troisième voie. Il faut concevoir une liberté politique qui se concilie, d'une part, avec la prise en compte du fait que. dans notre système politique, l'exécutif a la prépondérance - puisque les gouvernants ont l'initiative politique, ou les partis en tant qu'ils forment des gouvenants -, et d'autre part, avec le principe de la liberté individuelle. Paradoxalement, il me semble qu'on peut trouver un élément de réponse chez Rousseaau lui-même. Mais pas dans un contexte à proprement parler politique : dans le contexte d'un certain exercice de l'autorité pédagogique. C'est la situation dans laquelle se trouve l'adolescent qu'est Emile, au livre IV de l'ouvrage de Rousseau, de l'adolescent qui 
doit continuer d'être gouverné par son maître parce qu'il n'est pas encore capable de se fixer lui-même sa propre règle de conduite. Il est clair, pour Rousseau, que le pédagogue doit continuer à avoir l'initiative. Il doit être un gouverneur. Mais il faut essayer d'imaginer comment son élève, tout en n'ayant pas l'initiative, peut être encore libre et progresser vers l'autonomie. La solution qu'en donnne Rousseau est dans un contrat qui n'est pas du tout le contrat social et qui repose sur deux conditions. L'adolescent sera gouverné par le gouveneur, mais celui-ci s'engage à deux choses. Premièrement, il ne donnera à son élève que des ordres convenant à son bien objectif. Deuxièmement, il s'engage aussi à rendre rasion de son action. Il n'ordonnera rien qui ne puisse être contrôlé par le jugement de l'éduqué, rien qu'il ne s'engage à justifier par la suite. Non seulement il agira selon les raisons, mais il rendra raison de ses raisons. L'obéissance de l'individu dépend donc du fait que le gouverneur agit selon des raisons qu'il peut s'approprier. L'élève est libre parce que la volonté du gouverneur est une volonté qu'il peut faire sienne à partir du moment où il en saisit le principe. Ce qui est ordonné ne s'impose pas de l'extérieur à la volonté individuelle, puisque cette volonté, dès lors qu'elle pourra exercer son jugement et sa réflexion, pourra s'approprier l'ordre donné, comme si en quelque sorte elle le voulait ellemême. Ainsi la rationalité pédagogique que met en oeuvre Rousseau est une rationalité qui est à la fois objective et, si l'on peut dire, subjective. Elle est objective, puisqu'elle est censée corres-pondre au bien de l'éduqué. Mais elle est subjective, au sens où elle fait sa place au droit imprescriptible de la volonté individuelle de n'obéir qu'à ce qui se présente à elle sous la forme d'un principe rationnel de conduite.

Si l'on part de cet exemple proprement pédagogique pour envisager la question de la liberté politique, on arrive à conclure que la liberté individuelle dépend d'une autorité politique rationnelle au double sens qui vient d'être évoqué. La liberté suppose que les lois et les institutions ne s'imposent pas à ma volonté comme une réalité étrangère ou extérieure, mais qu'elles deviennent les miennes dans la mesure où je leur donne un sens. Par exemple, je suis libre si, étant instituteur, j'ai l'obligation de faire de l'éducation civique et je parviens à donner un sens a cette obligation. Ce qui suppose deux choses: d'une part, qu'elle ait du sens en elle-même, d'autre part, qu'elle ait du sens pour moi. Si cette éducation n'a aucun sens je ne suis pas libre, et si j'en fais sans la penser je ne suis pas libre non plus. Ma liberté dépend pour partie de ma capacité à penser ce qui m'est demandé, à discriminer ce qui est raisonnable et ce qui ne l'est pas, ce qui est sensé ou non, et à m'approprier la part de sens qu'il y a dans les obligations qui me sont faites. Telle est peut- 
être ce qu'on peut appeler la responsabilité de l'enseignant, qui dépend de sa culture et de la mise en oeuvre de cette culture pour penser sa pratique. Mais, d'une manière générale, on peut dire que la liberté dépend en premier lieu du fait que je vis dans un Etat de droit, c'est-àdire qui agit selon des raisons et qui fait sa place à l'autonomie du jugement individuel, mais aussi, en second lieu, de la culture juridique et politique qui permet aux citoyens de penser les institutions dans lesquelles ils vivent et d'en faire les leurs. Il dépend donc pour partie de l'école que les individus soient politiquement libre ou pas.

On peut naturellement voir dans cette thèse le principe d'une manipulation: l'individu aurait l'illusion de la liberté en trouvant des raisons de faire ce dont il ne peut se dispenser, c'es-à-dire de faire de nécessité vertu. La rhétorique des politiques a justement pour but d'assujettir les hommes en leur permettant d'entretenir de telles illusions. Mais il faut noter, cependant, que cette liberté politique résidant dans une certaine culture et l'exercice actif d'une réflexion sur les institutions n'est pas une liberté sans effet. Si l'individu n'est pas censé obéir sans comprendre et sans juger, cela signifie qu'un Etat qui agit selon des raisons absurdes ne peut pas exiger raisonnablement le respect des citoyens. L'individu peut donc refuser d'obéir à des ordres insensés, à des absurdités, à une politique qui serait contraire aux principes élémentaires du droit, au respect de la personne. De ce point de vue, la culture juridique et politique dont nous parlions peut et doit avoir pour effet de transformer l'idée de droit en force politique, c'es-à-dire de mettre la communauté en état d'opposer la résistance de l'idée de droit à l'action d'un gouvernement arbitraire.

Maintenant, si l'on accepte le principe d'une formation du jugement individuel au moyen d'une culture juridique et politique, il faut se poser la question du comment. A cette question, aucune réponse pleinement satisfaisante ne peut être donnée. On peut cependant s'appuyer sur les droits de l'homme, c'est-à-dire sur la fréquentation d'un certain nombre de textes où sont codifiés les principes sur lesquels l'Etat est censé reposer - la déclaration de 1789 a valeur constitutionnelle en France, elle peut donc servir de critère pour apprécier la valeur d'un projet de loi ou d'un programme politique. Autrement dit, on peut envisager une éducation civique sous la forme d'une éducation du jugement - bien partielle, parce que le jugement politique est quelque chose de plus complexe qu'une simple évaluation critique -, au moyen de la fréquentation des textes et par la manipulation des concepts qui permettent de formuler, d'expliciter, d'articuler les réalités sociales et politiques. 
Un certain nombre de questions de fond se posent ici qu'il faudrait résoudre pour pouvoir avancer. Il y en a au moins deux. Premierement, le fondement philosophique des droits de l'homme fait problème. En effet ce fondement est le droit naturel, et sans vouloir entrer dans les détails, on pourrait montrer que la notion de droit naturel, stricto sensu, est contradictoire. Qu'est-ce qu'un droit que l'individu posséderait par nature, c'est-à-dire indépendamment de l'appartenance à un Etat? Mais, d'un autre côté, on pourrait dire que les droits de l'homme sont suspects dans la mesure où ils representent les droits de l'individu isole. Parler des droits de l'individu isolé, c'est faire fi de son appartenance à des groupes, sociaux et à tout ce que cela peut impliquer d'inégalités. Mais ce que l'on peut chercher dans les droits de l'homme, si l'on veut en faire l'un des éléments de la formation d'une culture politique, ce n'est pas tant les droits naturels de l'individu isolé qu'une théorie de l'Etat, c'est-à-dire l'esquisse de cette rationalité politique à laquelle nous faisions allusion tout à l'heure: une rationalité à la fois objective, qui se veut cohérente, et en même temps qui implique, dans son propre fonctionnement, la part du jugement individuel. C'est en tant que théorie de l'Etat de droit qu'on peut utiliser les droits de l'homme comme critère du jugement et s'appuyer sur eux pour refuser un certain nombre de choses.

Bien entendu, cette théorie n'est pas satisfaisante. Il y bien des choses à redire: ce n'est pas le dernier mot de la philosophie politique et l'on peut exercer son jugement critique. Mais, peut-être est-ce un avantage. Car les droits de l'homme sont un critère nécessaire mais insuffisant. Insuffisant, notamment, parce qu'ambigu. Si on lit les articles $\mathrm{X}$ et XI de la déclaration de 1789 , par exemple, on voit que la liberté de communiquer et de manifester ses opinions doit s'exercer dans les limites de l'ordre public. Or, les limites de l'ordre public sont fixées par l'Etat, dans des termes qui laissent une large part à l'appréciation des gouvernants. Qui fixe la limite, où, comment, suivant quel critère? Les droits de l'homme posent donc des problemes. Mais c'est justement pour cela qu'ils nous intéressent. Ils posent la question des rapports entre l'individu et l'Etat, de l'exercice de l'autorité politique et de la part que cette autorité doit laisser au jugement individuel. Aussi peut-on dire deux choses, pour conclure. Les droits de l'homme nous donnent un critere qui permet de dire "non". Mais en même temps, parce qu'ils sont problématiques, ils nous donnent un critere qui ne peut pas se transformer en dogme, en bible, en religion, qui ne peut pas déboucher sur cet exercice mécanique du jugement qui consisterait en la simple application, "automatique", de tel ou tel article à telle ou telle situation. Bien sûr ils 
permettent de juger, mais si du moins on analyse les textes de près, ils mettent le jugement, non sur la voie ddu dogmatisme, mais sur celle de la réflexion. Ainsi y a-t-il peut-être moyen de tracer une voie pour l'éducation civique, entre le cathéchisme républicain et l'exaltation des bons sentiments.

PATRICE CANIVEZ 